\title{
SYNTHESIS OF THE LAWS GOVERNING THE NON- HOLONOMIC MODEL OF A TWO-LINK ROAD TRAIN WITH REVERSE MOTION (OFF-AXLE HITCHING MODEL)
}

\author{
Dmitry Tatievskyi \\ Department of Software of Automated Systems \\ Zaporizhzhia State Engineering Academy \\ 226 Soborny ave., Zaporizhzhia, Ukraine, 69006 \\ dtdissert@gmail.com
}

\begin{abstract}
The complexity of the control of the road train is due to the pronounced nonlinearities, as well as the instability of the control object during the movement in the backward motion (jackknifing). For the road trains, the location of the towing device behind the tractor's rear axle is quite typical. In this study, a synthesis of control laws for road trains with offset of coupling devices relative to the rear axle of the tractor (off-axle hitching) is proposed. The controllers have been implemented both to ensure a stable circular motion and for rectilinear motion with a given orientation angle, and the behavioral features of this model have been studied on the basis of them.

Based on the analysis of the approaches to the synthesis of the laws governing the road train with the coupling out, it was decided to synthesize the required control laws using the Lyapunov function method.

Synthesized controllers can be directly used to program the robotic systems of the respective models. It is also possible to use them for the development of the Dubins machine for the investigated model. They can be used to build automatic control systems that would help the driver to drive a car with a trailer while driving backward.

In this research, a study was made of the state of the solution of the problem associated with the reverse movement of a road train consisting of a tractor and a semitrailer with a coupling, synthesized laws made it possible to study the features of such model, determined by its linear dimensions.

For comparison of the synthesized laws, the analysis of phase portraits of trajectories, angles of folding and control, orientation angles was carried out, and also the analysis of the quality of transient processes with the change in the speed of the road train was performed.
\end{abstract} control law.

Keywords: road train, kinematic model, on-axle hitching, off-axis hitching, route curve, controller, non-holonomic system,

\section{Introduction}

Road trains consisting of a tractor and passive semitrailers belong to the class of nonlinear, non-holonomic, structurally unstable (with reverse motion) dynamic systems. Because of this, the task of controlling their movement is relevant both from the point of view of theory and from the point of view of the practical implementation of programmed motion with given constraints.

But, there is a problem of controlling such system when driving reverse (parking, entry into a limited space ("dock"), maneuvering when going back out of the dock, etc.). Without proper control action, the links of the road train will "squeeze out" each other, which is accompanied by an unlimited increase in the angle of folding. This phenomenon, known as jackknifing, while moving forward the semitrailer will track the tractor's heading angle. Therefore, autonomous cargo transportation systems are, at present, the object of numerous research projects, among which it is possible to single out works on the synthesis of control laws for the reverse movement of such systems.

The problem of controlling the movement of a road train with a semitrailer under the assumption of non-holonomic constraints (the absence of lateral slippage of the support wheels) is of great theoretical and practical importance. Research in this field is stimulated by numerous applied problems.

It should be noted that work in this direction is actively conducted abroad, especially in recent years. In this case, as a rule, separately considered models are "on-axle hitching" and "off-axle hitching", the control laws for which are synthesized by various methods. 
For the investigated model, management and planning methods are proposed using a diverse mathematical apparatus. Among the most frequently used methods, let's single out the method of feedback linearization [1, 2] and chain systems [3]. Due to the fact that such systems are differentially flat, the technique proposed by the authors of $[4,5]$ is well applicable to them.

Methods are also used, which are exclusively due to the geometric features of the kinematics of the "off-axle hitching" model, formulated in cascade form [6]. The apparatus of Lie algebras [7] and fuzzy logic [8] is also applied. A cascading control strategy (VFO) is used [6]. Linear quadrature controllers [9], nilpotent approximation [10], and so on are used.

A qualitative analysis of such systems is carried out in [11].

The main aim of research is synthesis of the laws governing the wheel module of the road train with the displacement of the coupling device relative to the tractor's rear axle, when driving with the reverse. This ensures the movement of the road train in the specified corridor, and is achieved by imposing restrictions on the values of the angles of folding between the links of the road train.

In applied works, based on the mathematical theory of control, the model of a controlled object, called the Dubins machine [12], is very popular. Such model is given by a nonlinear system of third-order differential equations. Two phase variables characterize the geometric position of the controlled object on the plane, the third variable angle of the direction of the velocity vector. The speed is considered constant. The scalar control action, constrained by the geometric constraint, determines the instantaneous turning radius. Obviously, in this capacity, it is possible to consider the proposed model, and solve for it the task of achieving a given aim.

In this paper, the synthesis of the control law is based on the Lyapunov function method [13]. It is possible to note that the direct Lyapunov method has already been successfully applied for the synthesis of a tractor control law with one semitrailer with a hitch in the "on-axle hitching" model [14].

Maple system is used for symbolic calculation of control laws, numerical integration and modeling. At the same time, the structure of the script in the internal language of the Maple system has a modular structure: the synthesis of the control law in symbolic form, the recording of the $\mathrm{CDS}$, the input of data, numerical integration, the mapping of phase portraits.

\section{Materials and methods}

At the heart of the mathematical model of the controlled motion of the road train, the classical positions of the mechanics of the system of solids lie in the reverse (taking into account the presence of non-holonomic constraints). The task of synthesizing programmed controlled motion was solved on the basis of rigorous approaches to the theory of automatic control and control of dynamical systems. The correctness of the obtained results is verified on the basis of independent numerical simulation of the system, namely:

1. The kinematic model of a three-link road train with non-holonomic constraints (no lateral slippage of the wheel system) with an off-axle hitching coupling model is considered.

2. To stabilize the movement of the reverse of such system, the Lyapunov function method is used (Lyapunov second method). The property of stability is considered from the point of view of the definition of Lyapunov stability.

3. When implementing the method, the necessary symbolic calculations are performed in the Maple environment.

4. Numerical simulation (integration) of the equations of controlled motion of the road train model is also carried out in the Maple environment.

The empirical study consists in realizing the controlled motion of a large-scale physical model, registering the angles of folding and controlling the impact, which allowed the optimal adjustment of the regulators for both synthesized control laws.

\section{Synthesis of control laws. Kinematic model of the system}

The movement of a system consisting of a tractor with a passive semi-trailer, connected by the "off-axle hitching" model, describes the single-track kinematic model quite accurately at low 
speeds. This model does not take into account the slip between the wheels and the roadway. Angular displacements in vertical planes are also not taken into account (Fig. 1).

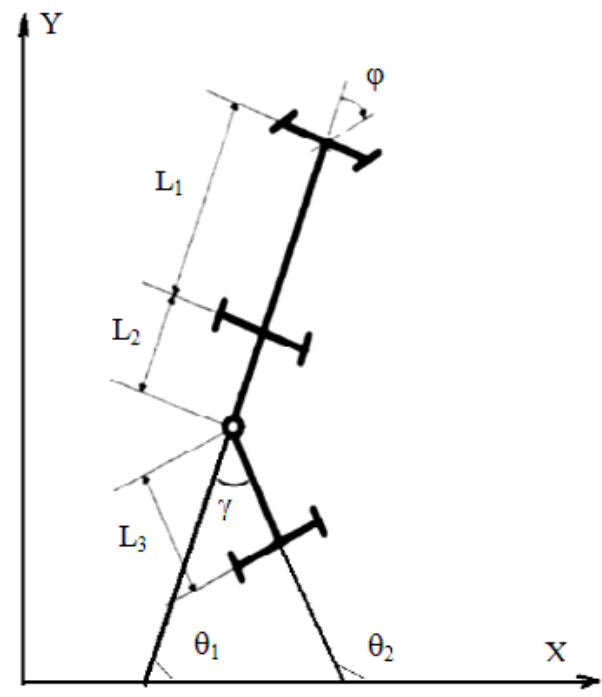

Fig. 1. The kinematic scheme of the system

Here $\theta_{1}, \theta_{2}$-the orientation angles of the system objects, $\mathrm{L}_{1}, \mathrm{~L}_{2}, \mathrm{~L}_{3}$ - their linear dimensions, $\varphi$ - the steering wheel angle of the tractor unit, $\gamma=\theta_{2}-\theta_{1}$ - the angle of folding between the tractor and the semitrailer. At this angle, a restriction is $|\gamma|<\pi / 2$, the restrictions on the angle $\varphi$ are determined by the technical control capabilities.

Such scheme is described by the following system of differential equations (1), obtained on the basis of the studies [15]:

$$
\begin{gathered}
\dot{\mathrm{x}}_{1}=\mathrm{v} \cdot \cos \left(\theta_{1}\right), \\
\dot{\mathrm{y}}_{1}=\mathrm{v} \cdot \sin \left(\theta_{1}\right), \\
\dot{\theta}_{1}=\mathrm{v} \cdot \tan (\phi) / \mathrm{L}_{1}, \\
\dot{\theta}_{2}=-\left(\mathrm{L}_{2} \cdot \dot{\theta_{1}} \cdot \cos (\gamma)+\mathrm{v} \cdot \sin (\gamma)\right) / \mathrm{L}_{3},
\end{gathered}
$$

where $\mathrm{v}<0$ - the linear speed of the tractor.

Compared with the system used to describe the on-axle hitching model, the latter equation contains the angular velocity of the tractor as a factor, complicating the synthesis of the control law. The same factor is also contained in the equation of trailer speed $v_{1}(2)$ [16]:

$$
\mathrm{v}_{1}=-\mathrm{L}_{2} \cdot \theta_{1} \cdot \sin (\gamma)+\mathrm{v} \cdot \cos (\gamma)
$$

which makes it difficult to control and stabilize the system.

Obviously, the first term on the right-hand side of (2) is determined by the rotational motion of the tractor (at $v=0$ ), and the second by the forward motion of the tractor (if its angular velocity is zero).

For numerical integration and modeling, the following design parameters are used: $\mathrm{L}_{1}=5$ $\mathrm{m}, \mathrm{L}_{2}=2 \mathrm{~m}, \mathrm{~L}_{3}=4 \mathrm{~m}$, Cauchy conditions of a rather general form $\theta_{1}(0)=-\pi / 10, \theta_{2}(0)=\pi / 15$ - zigzag, desired folding angle and orientation $\gamma_{\text {ref }}=\theta_{2 \text { ref }}=\pi / 5, \mathrm{v}=-1 \mathrm{~m} / \mathrm{s}$, the time intervals varied for clarity of phase portraits. Specifically, the change in these parameters is discussed when studying the behavioral features of this model. 


\section{Synthesis of a circular controller}

Let's define the tracking error $\mathrm{e}=\gamma_{\text {ref }}-\gamma$, where $\mathrm{y}_{\text {ref }}=$ const - the desired folding angle and choose the Lyapunov function (3) that satisfies the necessary conditions (4):

$$
\begin{gathered}
\mathrm{V}_{1}=\mathrm{e}_{1}^{2} / 2 . \\
\mathrm{V}(0)=0, \\
\mathrm{~V}(\mathrm{x}(\mathrm{t}))>0, \quad \mathrm{x} \neq \mathrm{x}_{0}, \\
\mathrm{~V}(\mathrm{x}(\mathrm{t})) \Leftrightarrow \mathrm{e} \cdot \dot{\mathrm{e}} \leq 0, \forall \mathrm{t}>0 .
\end{gathered}
$$

Its derivative $\dot{\mathrm{V}}=\mathrm{e} \cdot \dot{\mathrm{e}}=\mathrm{e} \cdot \dot{\gamma}$, since $\gamma_{\text {ref }}$ has a constant value.

In the Maple system, calculate the derivative for the folding angle $\gamma$, substituting the necessary expressions from the system (1), taking into account the relationship $\gamma=\dot{\theta}_{2}-\dot{\theta}_{1}$, Fig. 2.

$$
\begin{aligned}
& = \\
& >\operatorname{diffG}:=\operatorname{simplify}(\text { diffTheta2 }-\operatorname{diffTheta} 1) ; \\
& =\quad \operatorname{diffG}:=-\frac{\left.\left(\mathrm{L}_{1} \sin (\mathrm{g}(\mathrm{t})) \cos (\phi)+\sin (\phi)(\cos (\mathrm{g})(\mathrm{t})) \mathrm{L}_{2}+L_{3}\right)\right) V}{\cos (\phi) \mathrm{L}_{1} \mathrm{~L}_{3}}
\end{aligned}
$$

Fig. 2. The derivative of the folding angle

The control law is obtained from the condition of its negative semidefinite, assuming its value is equal to ke, where $\mathrm{k}>0$ and, solving this equation with respect to control $\varphi$, let's obtain Fig. 3 .

$$
\begin{aligned}
& = \\
& >\mathrm{E}_{x}:=\operatorname{diffG}-k \cdot e ; \\
& E_{x}:=-(g \operatorname{Re} f-g(t)) k-\frac{\left.\left(\mathrm{L}_{1} \sin (\mathrm{g}(\mathrm{t})) \cos (\phi)+\sin (\phi)(\cos (\mathrm{g})(\mathrm{t})) \mathrm{L}_{2}+L_{3}\right)\right) V}{\cos (\phi) L_{1} L_{3}} \\
& = \\
& >\text { Phi }:=\operatorname{simplify}\left(\operatorname{solve}\left(\mathrm{E}_{x}=0, \text { phi }\right)\right) ; \\
& \Phi:=-\arctan \left(\frac{\left.\mathrm{L}_{1}(\mathrm{~V} \sin (\mathrm{g}(\mathrm{t})))+\mathrm{kL}_{3}(\mathrm{gRef}-\mathrm{g}(\mathrm{t}))\right)}{\mathrm{V}\left(\cos (\mathrm{g}(\mathrm{t})) \mathrm{L}_{2}+\mathrm{L}_{3}\right.}\right)
\end{aligned}
$$

Fig. 3. Synthesis of the control law

Consequently, the circulation controller has the form (4):

$$
\phi=-\operatorname{arctg}\left(\left(\mathrm{L}_{1} \cdot \mathrm{v} \cdot \sin () \gamma+\mathrm{k} \cdot \mathrm{L}_{3} \cdot \mathrm{e}\right) /\left(\mathrm{L}_{2} \cdot \mathrm{v} \cdot\left(\cos (\gamma)+\mathrm{L}_{3}\right)\right)\right)
$$

The derivative of the Lyapunov function, however, is negative semidefinite, which ensures the asymptotic stability of the circular motion of the system, and the synthesized controller can be directly used for program control of the investigated system.

Based on the results of numerical simulation, the obtained phase portraits present in the figures below (Fig. 4-6).

In this case, before the beginning of the program motion with a given folding angle, it is possible to calculate the curvature radius of the circular trajectory by solving equation (5) [15] with respect to $\mathrm{R}$, taking its norm (6):

$$
\begin{gathered}
\gamma_{\text {ref }}=\pi-\arctan \left(\mathrm{R} / \mathrm{L}_{2}\right)-\arccos \left(\mathrm{L}_{3}^{2} /\left(\mathrm{R}^{2}+\mathrm{L}_{2}^{2}\right)^{1 / 2}\right), \\
\mathrm{R}=\left|\left(\mathrm{L}_{2} \cdot \cos \left(\gamma_{\text {ref }}\right)+\mathrm{L}_{3}\right) / \sin \left(\gamma_{\text {ref }}\right)\right|
\end{gathered}
$$




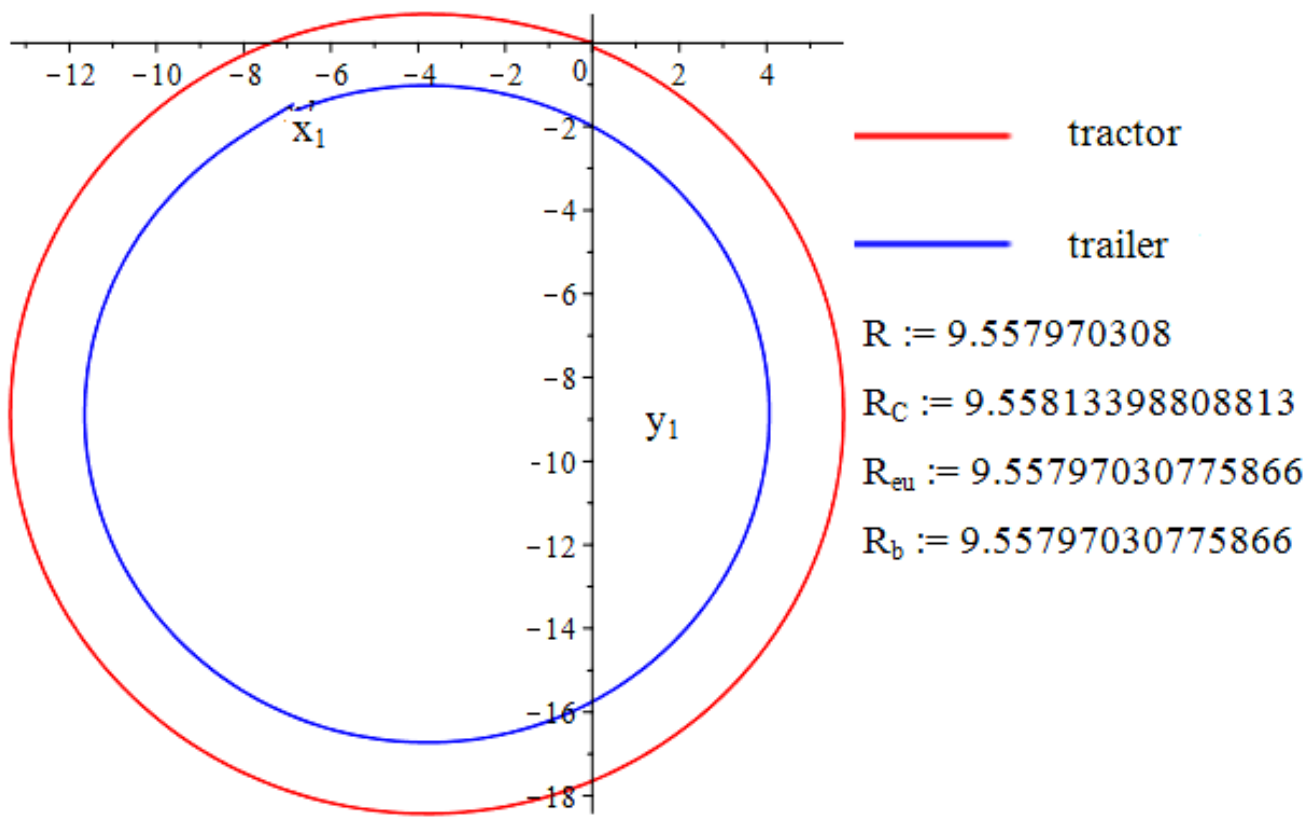

Fig. 4. Trajectories of circular movement

The value of $\mathrm{R}$ calculated in this way practically coincides with the values of the radii calculated from the results of numerical integration. The values of the radii in Fig. 4 calculated from the coordinates of the trajectory $\left(R_{c}\right)$, according to the Euler formula $\left(R_{e u}\right)$, using the control value $\left(\mathrm{R}_{\mathrm{u}}\right)$. For the last three formulas, the last values in the arrays generated by the Maple tools for phase portraits are used (Fig. 5).

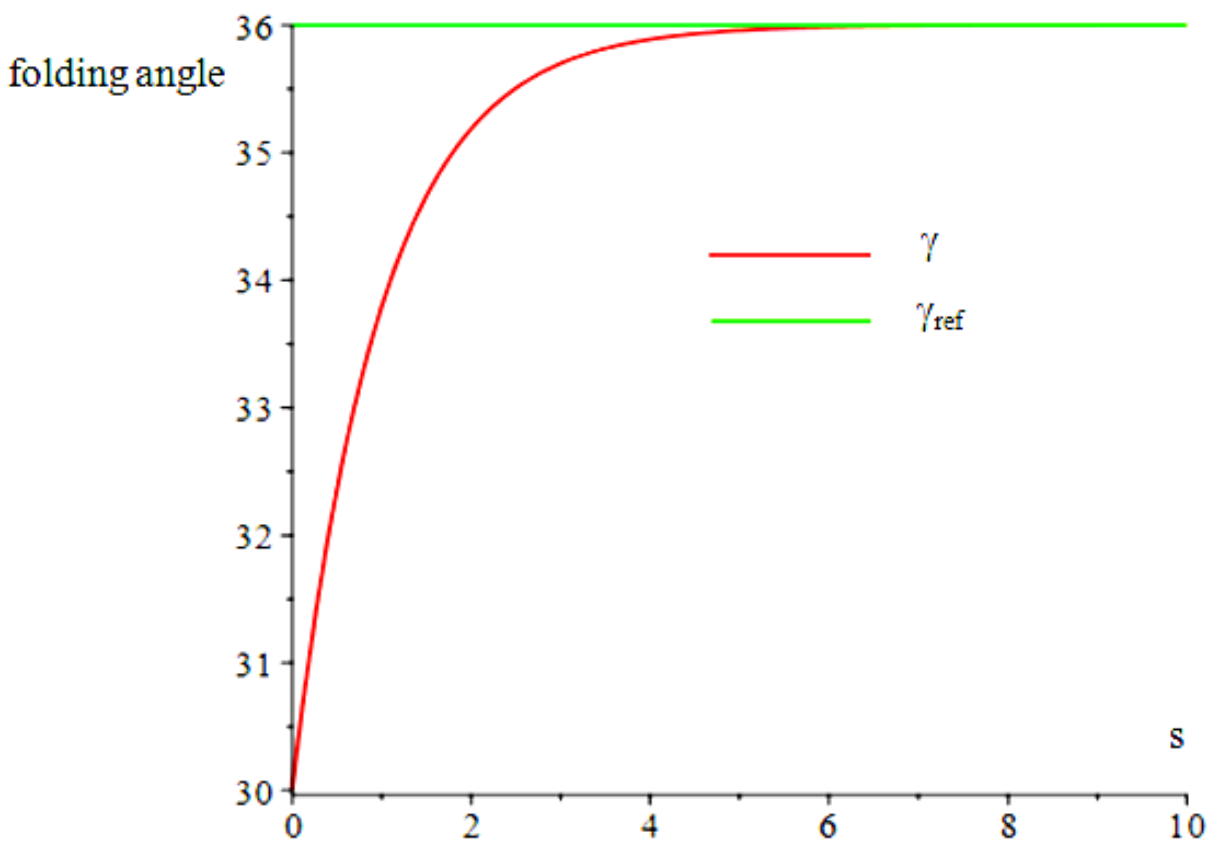

Fig. 5. Dynamics of the folding angle with reverse curvilinear motion

Also, it is possible to calculate the asymptotic value of the control using the radius of the circle R calculated above (trajectory of the track) $\phi_{\text {ref }}=\arctan \left(\mathrm{L}_{1} / \mathrm{R}\right)$, and display it on the phase portrait (Fig. 6).

In this case, the control coefficient $\mathrm{k}$ in the control law was chosen to be 1.4 , which made it possible to optimally adjust the control value and the transient time. 


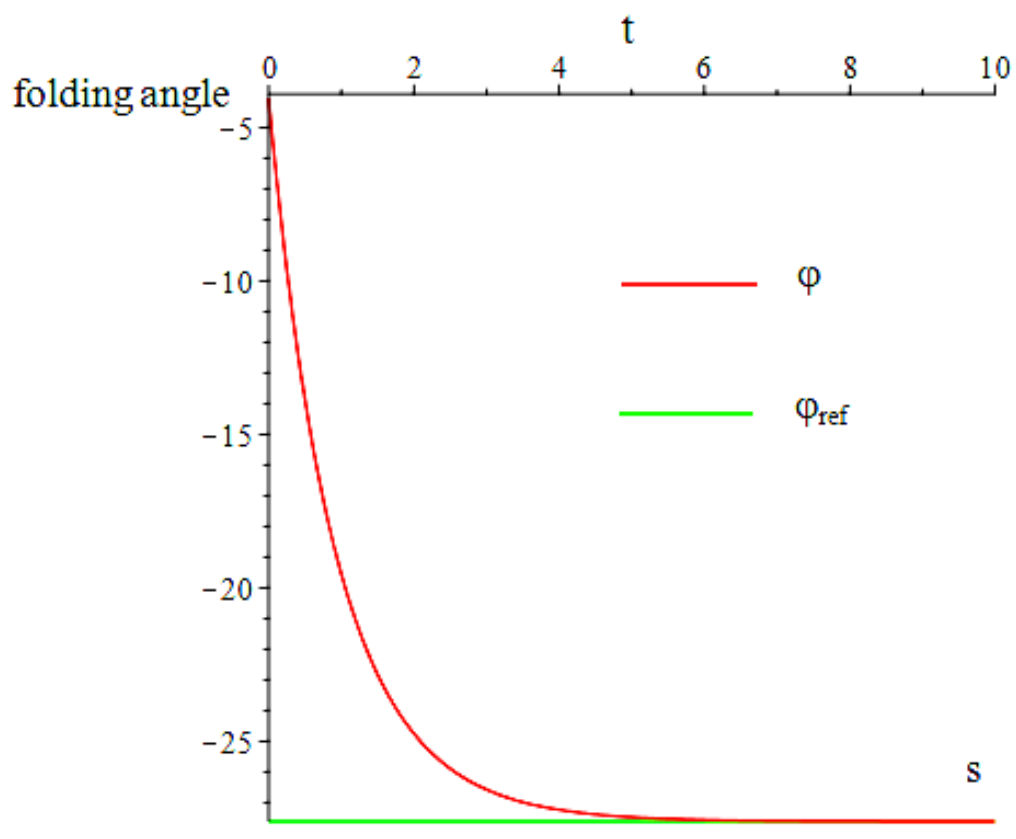

Fig. 6. Dynamics o of the road train control

\section{Synthesis of the orientation controller}

The control law for this controller (motion with a given orientation angle) is synthesized on the basis of the previous law, selecting the tracking error $\mathrm{e}=\gamma_{\text {ref }}(\mathrm{t})-\gamma(\mathrm{t})$ and demanding the condition $\gamma_{\text {ref }}(t)=\theta_{2 \text { ref }}-\theta_{2}$, where $\theta_{2 \text { ref }}=$ const - desired orientation angle of the semitrailer.

The derivative of the Lyapunov function (2) (Fig. 7):

$$
\begin{aligned}
>\text { diffG }:=\operatorname{simplify}(\text { diffTheta } 2-\text { diffTheta1 }) \\
\qquad \text { diffG }:=-\frac{\left.\mathrm{V}\left(\mathrm{L}_{1} \sin (\mathrm{g}(\mathrm{t})) \cos (\phi)+\sin (\phi)(\cos (\mathrm{g})(\mathrm{t})) \mathrm{L}_{2}+L_{3}\right)\right)}{\cos (\phi) \mathrm{L}_{1} \mathrm{~L}_{3}}
\end{aligned}
$$

Fig. 7. The derivative of the folding angle

From the condition of negative semidefinite (assuming its value is equal to ke, where $\mathrm{k}>0$ ), the control law follows (Fig. 8) (7):

$$
\begin{aligned}
& >\mathrm{E}_{\mathrm{x}}:=\operatorname{diff} G-k \cdot e ; \\
& >\text { Phi }:=\operatorname{simplify}\left(\operatorname{solve}\left(\mathrm{E}_{\mathrm{x}}=0, \mathrm{phi}\right)\right) ; \\
& \Phi:=-\arctan \left(\frac{\left.\mathrm{L}_{1}(\mathrm{~V} \sin (\mathrm{g}(\mathrm{t})))-\mathrm{kL}_{3}(\mathrm{~g}(\mathrm{t})-\text { theta2Ref }+\theta 2(\mathrm{t}))\right)}{\mathrm{V}\left(\cos (\mathrm{g}(\mathrm{t})) \mathrm{L}_{2}+\mathrm{L}_{3}\right.}\right)
\end{aligned}
$$

Fig. 8. Synthesis of the control law

$$
\begin{aligned}
& \text { phi }=-\arctan \left(\left(\mathrm{L}_{1} \cdot(\mathrm{v} \cdot \sin (\gamma(\mathrm{t}))-\right.\right. \\
& \left.\left.\left.-\mathrm{k} \cdot \mathrm{L}_{3} \cdot\left(\gamma(\mathrm{t})-\theta_{2 \text { ref }}+\theta_{2}(\mathrm{t})\right)\right)\right) /\left(\mathrm{v} \cdot\left(\cos \left(\gamma(\mathrm{t}) \cdot \mathrm{L}_{2}+\mathrm{L}_{3}\right)\right)\right)\right) .
\end{aligned}
$$

For the synthesized orientation controller, the phase portraits presented in the figures below are obtained (Fig. 9-12).

In this case, the value of the orientation angle computed along the path of the phase portrait

$$
\theta=\arctan (\delta \mathrm{Y} / \delta \mathrm{X})=35.9999948305423,
$$

at a specified desired angle of $36^{\circ}$. 


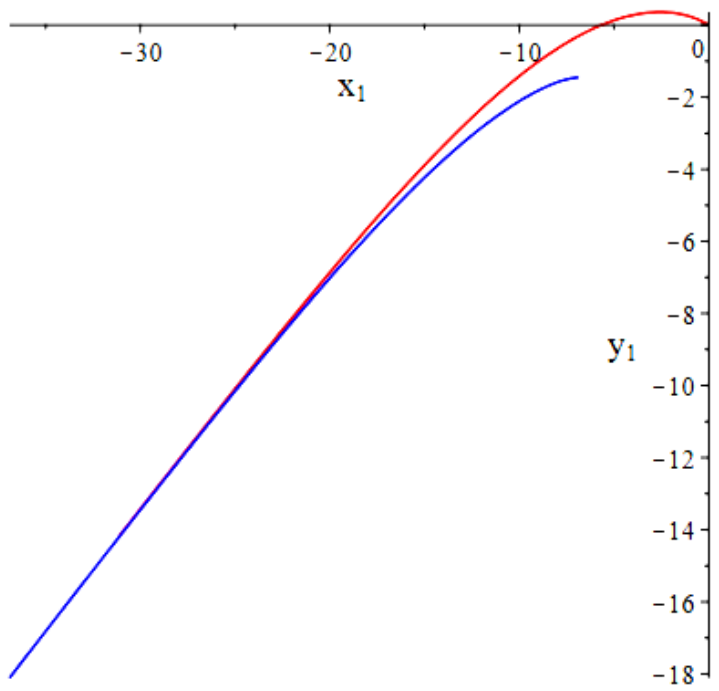

Fig. 9. Trajectory of tractor and trailer movement

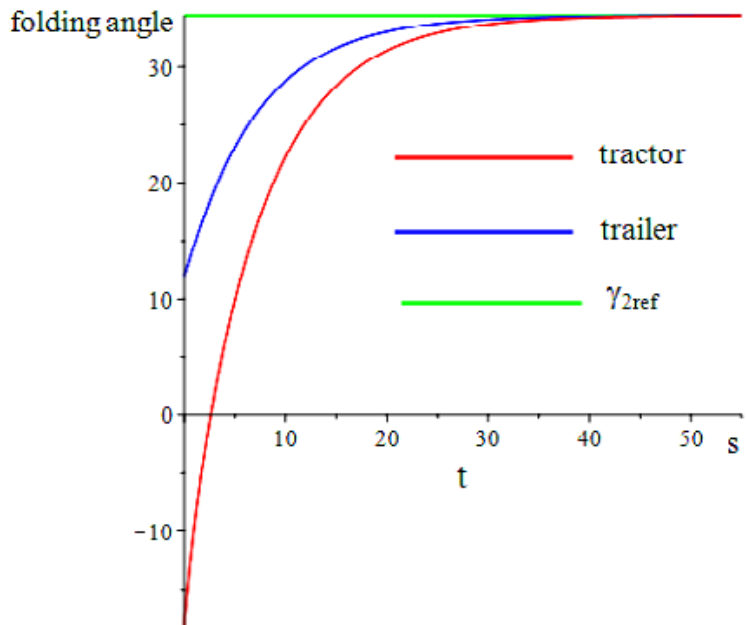

Fig. 10. Orientation angles of tractor and trailer

Control dynamics.

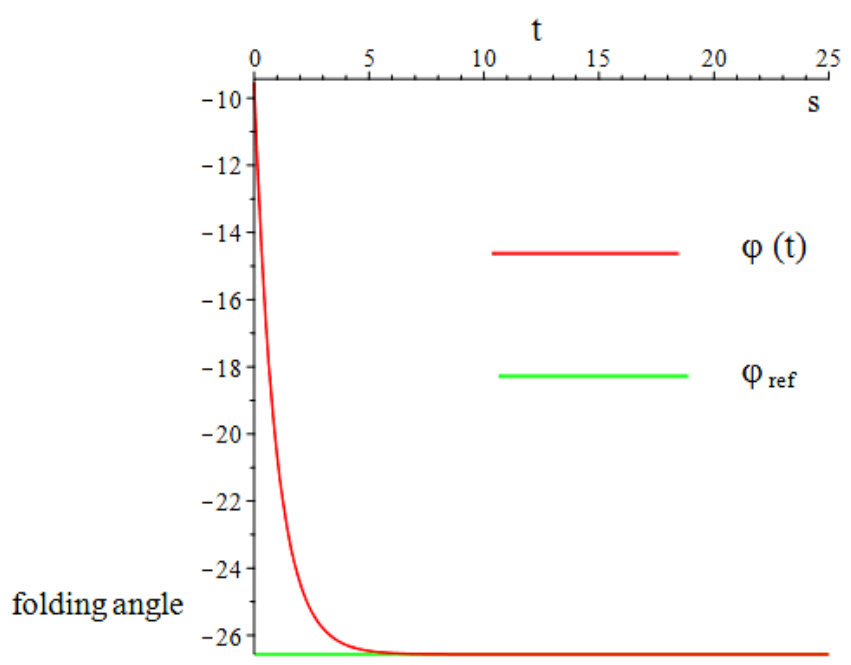

Fig. 11. Steering wheel deflection angle (control) 
The folding angle (blue) and the control (red) in this case tend to zero (Fig. 12).

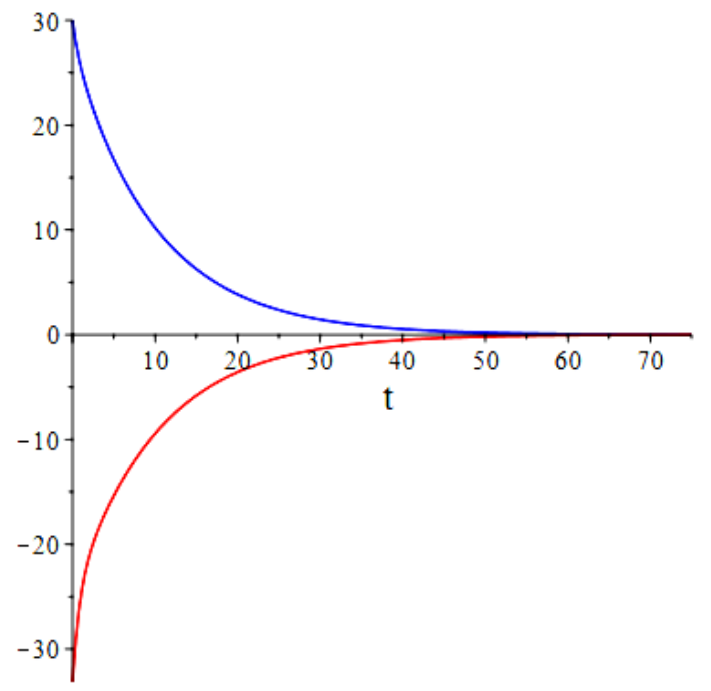

Fig. 12. Dynamics of the folding and control angles

\section{Synthesis of the law for realizing the movement of a road train on a given route curve}

As is known, two types of tracking automatic control systems are used for driving vehicles: a "trajectory tracking" system and a control system for "path following".

In the first case, the trajectory specifies the desired change in state variables as a function of time. In the second case, the desired route curve (a combination of lines and arcs of a circle of radius $\mathrm{R}$ ) in the workspace is set, which is not a function of time.

The laws synthesized above successfully solve the first problem. To solve the second, let's use the dependence (5), calculating the desired folding angle over a given radius of curvature.

When a radius value is received at the input of the Maple script, the desired folding angle is computed and the motion along the circumference (Fig. 13) occurs according to the law synthesized for the circular controller.

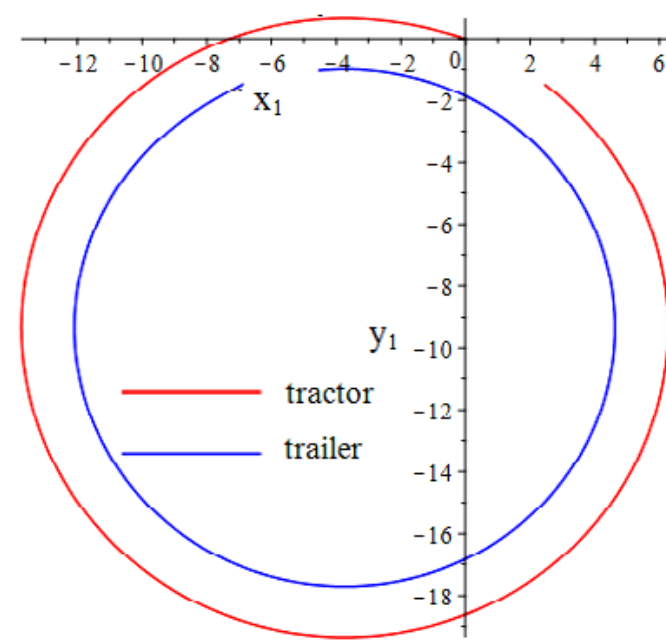

$$
\begin{aligned}
& \mathrm{R}:=10 \\
& \mathrm{R}_{\mathrm{C}}:=9.99813052591851 \\
& \mathrm{R}_{\mathrm{eu}}:=9.99999999999981 \\
& \mathrm{R}_{\mathrm{u}}:=9.99999999999980
\end{aligned}
$$

Fig. 13. Movement along a curve of a given radius

The curves in Fig. 13 are the values of the radii, calculated in various ways: on the coordinates of the trajectory $\left(\mathrm{R}_{\mathrm{c}}\right)$, according to the Euler formula $\left(\mathrm{R}_{\mathrm{eu}}\right)$ and taking into account the control $\left(\mathrm{R}_{\mathrm{u}}\right)$. For the last three formulas, the last values of the arrays generated by the Maple tools for phase portraits were used. In this case, the value of the angle calculated from (5) is equal to $\gamma_{\text {ref }} 34.4^{\circ}$. 
Dynamics of control when using this law is shown in Fig. 14, the asymptotic value of the control is calculated by the formula $\phi_{\text {ref }}=a \tan \left(\mathrm{L}_{1} / \mathrm{R}\right)$.

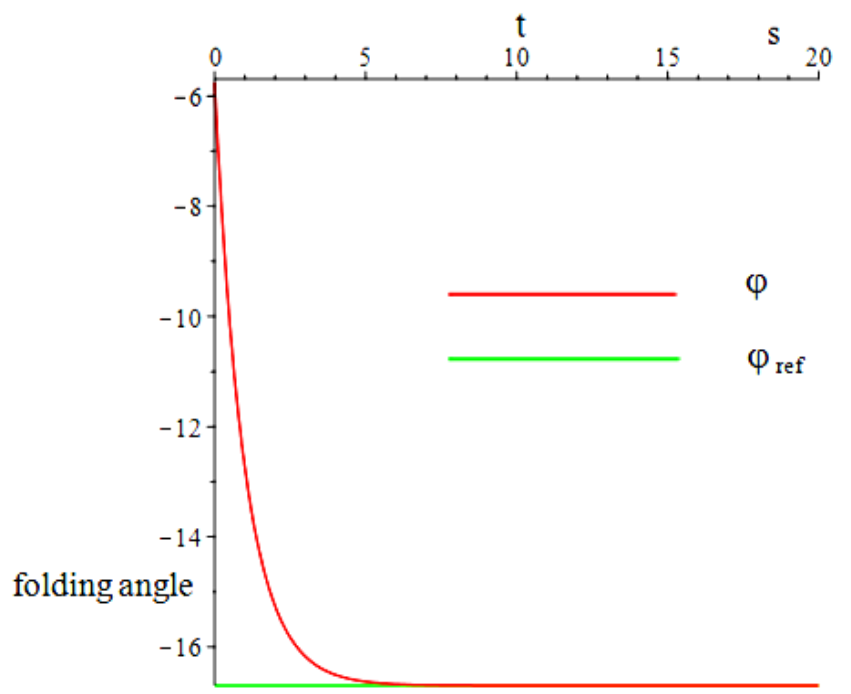

Fig. 14. Dynamics of control when driving on a given route curve

In this case, it should be borne in mind that in the case of $\mathrm{R}->\infty$ or $\gamma_{2 \text { ref }}->0$ the movement of the road train will tend to rectilinear.

\section{Investigation of the off-axle hitching model features}

Let's use synthesized controllers to study the features of this model. Changing its geometry - linear dimensions, it is possible to draw the following conclusions.

A decrease in the parameter $\mathrm{L}_{2}\left(\mathrm{~L}_{3}=\right.$ const) leads to an increase in control (Fig. 15), which reaches its maximum in the limiting case $\mathrm{L}_{2}=0$ (on-axle hitching model).

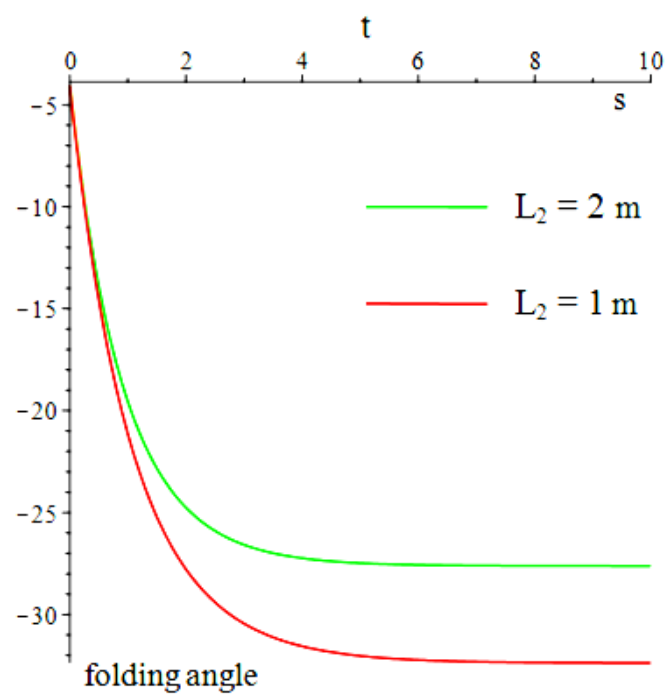

Fig. 15. Increase in the amount of control when the coupling decreaes

With an increase in the length of the semitrailer with a constant length of the coupling, the amount of control decreases (Fig. 16) and vice versa.

In this case, the best stability of the movement, in the sense of the speed of the trailer, is ensured when L2=L3, the semitrailer and tractor track a single trajectory (Fig. 17). 


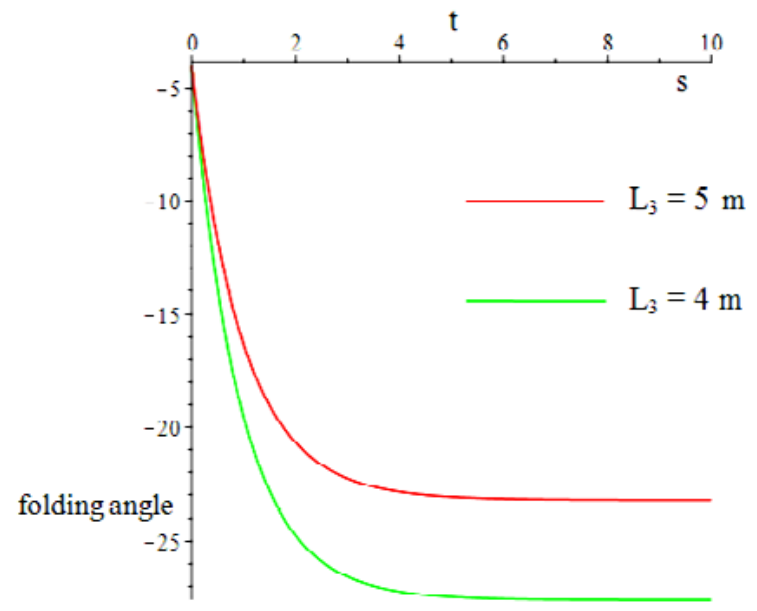

Fig. 16. Decrease in the amount of control as the length of the semitrailer increases

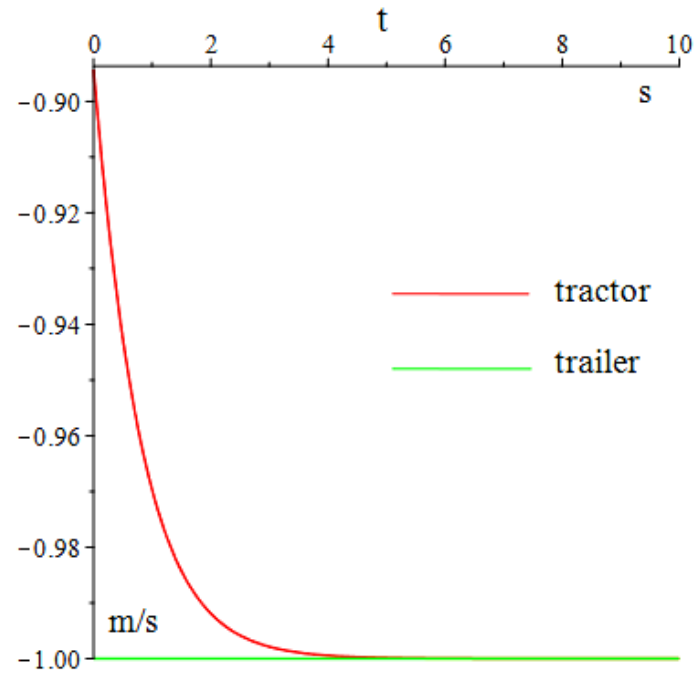

Fig. 17. Optimum stability of motion

Trailer speeds for other values of the length of the couplings (large and smaller than $4 \mathrm{~m}$ ) are shown in Fig. 18.

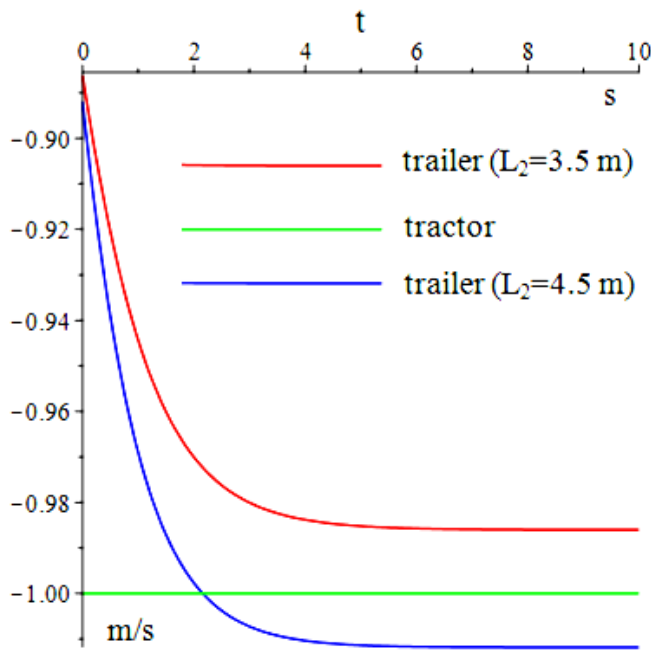

Fig. 18. Dynamics of trailer speeds for different coupling lengths (curvilinear motion) 
For rectilinear motion (using the orientation controller and circular) with the condition or, changing the linear dimensions of the system has practically no effect on the amount of control. At the same time, the speed of the semitrailer maximally tends to the tractor speed (Fig. 19).

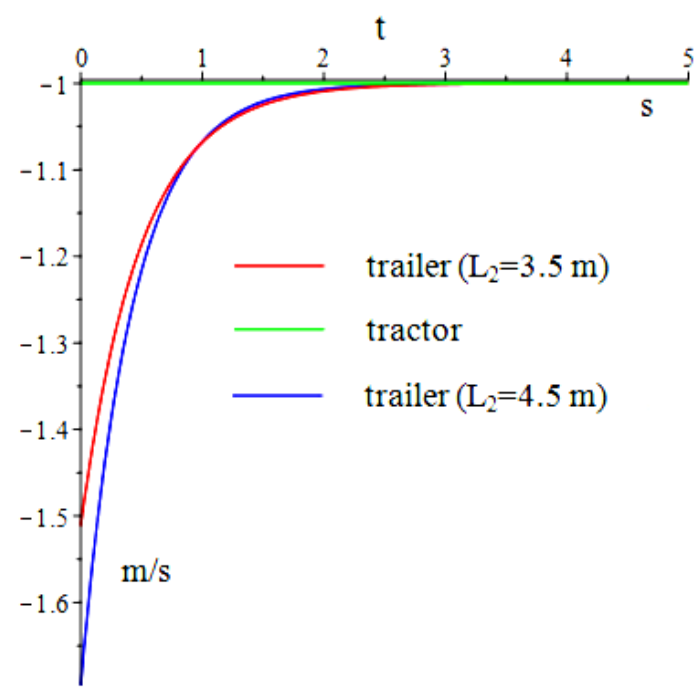

Fig. 19. Dynamics of orientation controller speeds

Thus, synthesized controllers can be useful in the development of robotic systems for their compliance with the necessary technological requirements.

\section{Conclusions}

1. A review of the existing complexes of theoretical and empirical methods is performed, the combination of which makes it possible to study the object with the greatest certainty in order to select the optimal mathematical apparatus for achieving the goal of synthesizing control laws for the reverse motion of a road train with coupling out.

2. The synthesis of such laws using the Lyapunov function method and using the Maple system for performing this synthesis, intermediate calculations and numerical integration of a system of differential equations is realized.

3. A control system has been synthesized for following a road train along a route curve defined by its curvature radius.

4. The features of the off-axle hitching model, determined by the ratio of its linear dimensions and the conditions for its maximum stability, are investigated.

5. A special feature of the study is that, unlike the approaches to synthesizing control laws for the studied model, the Lyapunov function method is used, we are not aware of the work on the synthesis of the orientation controller. The synthesized orientation controller now allows solving the problem of achieving the target by a road trains along the optimal trajectory within a certain reachable range without the use of switching. The efficiency of the method of Lyapunov functions will allow it to be used in the future in the backstepping method to solve the problem of reverse motion of road trains with an increasing number of links.

The practical value of this research is that synthesized control laws can now be applied for the programmatic management of the robot train. Such robotic system is under implementation. To control it, it is planned to integrate the synthesized controllers into a controller developed according to fuzzy logic.

In addition, the subject of further research will be the development of the Dubins machine based on synthesized control laws to ensure a stable rectilinear motion, with a given orientation angle and curvilinear, with a given radius to solve the problem of achieving the aim. 


\section{References}

[1] Bolzern, P., DeSantis, R. M., Locatelli, A., Masciocchi, D. (1998). Path-tracking for articulated vehicles with off-axle hitching. IEEE Transactions on Control Systems Technology, 6 (4), 515-523. doi: 10.1109/87.701346

[2] Altafini, C. (2003). Path following with reduced off-tracking for multibody wheeled vehicles. IEEE Transactions on Control Systems Technology, 11 (4), 598-605. doi: 10.1109/tcst.2003.813374

[3] Murray, R. M., Sastry, S. S. (1993). Nonholonomic motion planning: steering using sinusoids. IEEE Transactions on Automatic Control, 38 (5), 700-716. doi: 10.1109/9.277235

[4] Fliess, M., Levine, J., Martin, P., Rouchon, P. (1995). Design of trajectory stabilizing feedback for driftless flat systems. Proc. 3rd European Control Conf. Rome, 1882-1887.

[5] Rouchon, P., Fliess, M., Levine, J., Martin, P. (1993). Flatness and motion planning: the car with $\mathrm{n}$ trailers. Proceedings of the 32nd IEEE Conference on Decision and Control. Available at: http:// cas.ensmp.fr/ rouchon/publications/PR1993/ECC93.pdf

[6] Michalek, M. (2012). Application of the VFO method to set-point control for theN-trailer vehicle with off-axle hitching. International Journal of Control, 85 (5), 502-521. doi: 10.1080/00207179.2012.658524

[7] Sklyarenko, E. G., Shumakher, V. (2010). Stabilizatsiya zadnego khoda avtopoezda s dvukhosnym pritsepom metodom linearizatsii obratnoy svyaz'yu po sostoyaniyu. Visn. Nats. tekhn. un-tu «KHPI», 28, 275-278.

[8] Cheng, J., Wang, B., Zhang, Y., Xu, Y. (2016). Backward Path Tracking Control for Mobile Robot with Three Trailers. China, 32-41. doi: 10.1007/978-3-319-70136-3_4

[9] Evestedt, N., Ljungqvist, O., Axehill, D. (2016). Motion planning for a reversing general 2-trailer configuration using Closed-Loop RRT. 2016 IEEE/RSJ International Conference on Intelligent Robots and Systems (IROS), 3690-3697. doi: 10.1109/iros.2016.7759544

[10] Ardentov, A. A. (2016). Controlling of a mobile robot with a trailer and its nilpotent approximation. Regular and Chaotic Dynamics, 21 (7-8), 775-791. doi: 10.1134/s1560354716070017

[11] Martynyuk, A. A., Nikitina, N. V. (2018). O kachestvennom analize odnoy modeli transportnykh mashin. Prikladnaya mekhanika, 2, 110-115.

[12] Dubins, L. E. (1957). On Curves of Minimal Length with a Constraint on Average Curvature, and with Prescribed Initial and Terminal Positions and Tangents. American Journal of Mathematics, 79 (3), 497-516. doi: 10.2307/2372560

[13] Lyapunov, A. M. (1956). Collected Papers. Vol. 2. Moskow: Izd. Akd. Nauk SSSR, 472.

[14] Tatievskyi, D. (2017). The synthesis of steering rules for stabilizing road train reverse motion to solve the task of reaching a set goal. Technology Transfer: Fundamental Principles and Innovative Technical Solutions, 37-39. doi: 10.21303/2585-6847.2017.00482

[15] Cariou, C., Lenain, R., Thuilot, B., Berducat, M. (2010). Autonomous maneuver of a farm vehicle with a trailed implement: motion planner and lateral-longitudinal controllers. 2010 IEEE International Conference on Robotics and Automation. Anchorage. doi: 10.1109/robot.2010.5509447

[16] Lee, J.-H., Chung, W., Kim, M., Song, J.-B. (2004). A Passive Multiple Trailer System with Off-axle Hitching. International Journal of Control, Automation, and Systems, 2 (3), 289-297. 